\title{
Application of One-way coupled Hydro-meteorological Model in the Jinshajiang River Basin, China
}

\author{
Hairong Zhang, , a, Jianzhong Zhou', b, Xiaofan Zeng', c, Yi Liu', d, Jun Guo 2, 3, e \\ ${ }^{1}$ School of Hydropower and Information Engineering, Huazhong University of Science and \\ Technology, Wuhan 430074, China \\ 2. State Key Laboratory of Disaster Prevention and Reduction for Power Grid Transmission and \\ Distribution Equipment \\ 3. State Grid Hunan Electric Power Company Disaster prevention and reduction center \\ azhr@hust.edu.cn, bjz.zhou@hust.edu.cn, czengxiaofan2009@gmail.com, dformoon@foxmail.com, \\ e332413817@qq.com
}

\begin{abstract}
Keywords: the WRF model; the Jinshajiang River Basin; hydro-meteorological model
Abstract. In this study, a mesoscale weather model suitable for precipitation simulation in the Jinshajiang River Basin (the largest hydropower base in China) were firstly investigated. The results show that the WRF model could be used for precipitation simulation and prediction in the Jinshajiang River Basin. On the basis of the WRF model for precipitation in the Jinshajiang River Basin, a one-way coupled hydro-meteorological model was built and also achieved a good performance, which allows it to be used in hydrological forecasting operations to provide a forecast flood with high precision.
\end{abstract}

\section{Introduction}

Establishing a hydro-meteorological model for precipitation and runoff simulations that is suitable for specific basins is very important because it could help improve the precision of precipitation and runoff prediction for a given region. The prediction accuracy of precipitation-runoff processes can be improved to provide beneficial information for scheduling and utilization of the water resources in the basin at the same time.

Located in the upper reaches of the Yangtze River Basin, the Jinshajiang River Basin is the largest hydropower base in China. Belonging to the Hengduan Mountain region, the Jinshajiang River Basin has complex geographical and topographical conditions and is influenced by both the southeast and southwest monsoons and the Qinghai-Tibet Plateau. Because it is located in a transition region between the plateau and East China plain, the eastern region of the Qinghai-Tibet Plateau has an extremely complicated climate resulting from the influences of tropical, subtropical and plateau monsoons[1]. In recent years, global and regional warming has produced frequent floods and droughts in the Yangtze River Basin, which is where the Jinshajiang River Basin is located. The possibility of floods in the summer season and droughts in the fall is increasing[2]. For instance, there has been a noticeable increase of extreme precipitation-runoff events in the Jinshajiang River Basin, with the occurrence of the precipitation peak shifting to as early as June, which nearly coincides with the occurrence of the precipitation peak for the middle and lower reaches of the Yangtze River basin. This shift certainly increases the chance of floods in the Yangtze River Basin[3].

The Weather Research and Forecasting (WRF) model, known as a next-generation mesoscale numerical weather prediction model, offers a flexible and computationally-efficient platform for operational forecasting, taking advantage from recent advances in physics and numeric. It proved to be a useful tool in precipitation forecast and has proved to achieve some good results[4,5].

In this study, the Jinshajiang River Basin was used as the study area. Firstly the WRF model suitable for the Jinshajiang River Basin were investigated. Secondly the Xinanjiang model was brought herein as the hydrological model. The Jinshajiang River Basin was divided into several sub-basins and a forecasting system was built by establishing the Xinanjiang model in every sub-basin. The outlet flood forecasts can be obtained through the forecasting system using the WRF's 
precipitation as input. At last a one-way coupled hydro-meteorological system was built to generate precipitation and flood forecasts for the specified precipitation and flood events.

\section{Study Area and Research Methods}

Study Area. Located in the upper reaches of the Yangtze River Basin, the Jinshajiang River Basin is situated in the Qinghai-Tibet Plateau, the Yunnan-Guizhou Plateau and the western margin of the Sichuan Basin. The Jinshajiang River Basin has a catchment area of $470000 \mathrm{~km}^{2}$, and the main river of the Jinshajiang River has a length of $3496 \mathrm{~km}$ (from the source to Yibin, Sichuan). The Jinshajiang River flows through five terrain units, including the Qinghai-Tibet Plateau, the western Sichuan Plateau, the Hengduan Mountains, the Yunnan-Guizhou Plateau and the mountainous area of southwest Sichuan. The landforms and the topographical patterns of the Jinshajiang River Basin are extremely complicated, with the terrain showing a dramatic elevating trend from the southeast to northwest[6]. The north and the northwest of the Jinshajiang River Basin are vast plateaus, while the south and the southeast are canyons, which have a relative relief of 1000 to $3000 \mathrm{~m}$.

There are various climate types in the basin, which include typical plateau climates, stereoscopic climates and monsoon climates. The mean annual precipitation in the Jinshajiang River Basin is approximately $756.4 \mathrm{~mm}$. However, the precipitation has noticeable seasonal and regional variations, with higher precipitation in the lower reaches of the basin than in the upper reaches. The precipitation reaches its peak in the summer season. The mean annual river discharge at the hydrological station Xiangjiaba, which is located at the lower reaches of the basin, is approximately $4610 \mathrm{~m}^{3} / \mathrm{s}$. The river discharge through a year has a single-peak distribution, and its highest levels occur between July and September, a month after the annual precipitation peak.

Moreover, the Jinshajiang River Basin has a huge potential for hydroelectric energy and is the largest hydropower base in China, ranking first among the proposed 13 hydropower bases in China. It is the main contributing hydropower base of the "power transmission from west to east" project, and the hydroelectric energy reserves in the Jinshajiang River Basin are approximately 0.1124 billion $\mathrm{kw}$. Fig. 1 shows the geographical location of the Jinshajiang River Basin in China.

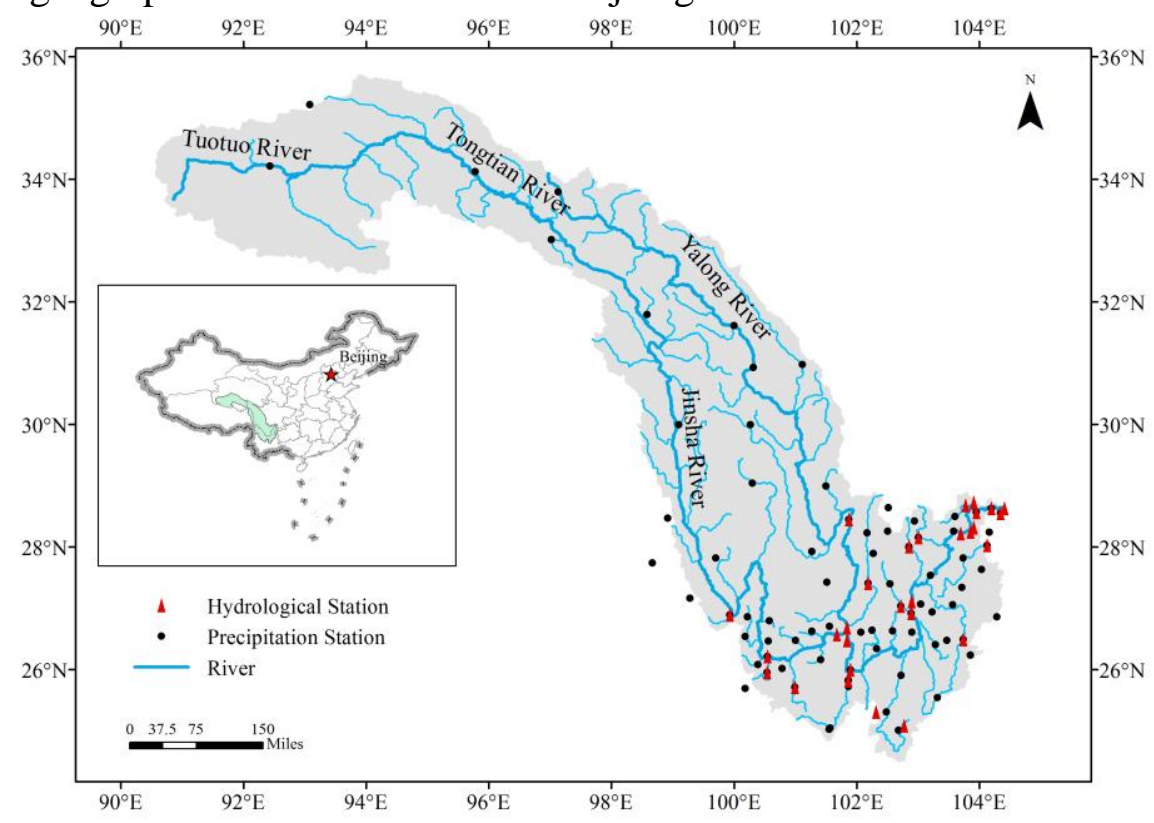

Fig. 1 The geographical location of the Jinshajiang River Basin in China.

The WRF Model. The WRF model is numerical weather prediction system designed for atmospheric research and weather forecasting and was collectively developed by a number of US research and development organizations, including the National Center for Atmospheric Research, the National Oceanic and Atmospheric Administration, the Forecast Systems Laboratory, the Center for Analysis and Prediction of Storms of University of Oklahoma[7]. 
In the present study, the WRF model was used to establish the numerical weather model for simulating precipitation in the Jinshajiang River Basin. A non-hydrostatic, fully compressible Euler equation was used as the dynamic framework. The terrain-following hydrostatic-pressure coordinate was used as the vertical coordinate, and the variables were staggered in the horizontal Arakawa $\mathrm{C}$-grid. The time scheme was the third order Runge-Kutta. In the vertical direction, $\eta$ area was used and divided into 27 layers. Based on the weather and climate characteristics of the Jinshajiang River, the rapid radiative transfer model (RRTM) scheme was used as the long-wave radiation scheme; the Goddard scheme was used as the short-wave radiation scheme; the Eta similarity scheme was used as the surface layer scheme; and the Noah scheme was used as the land surface process scheme; the Lin et al. scheme was used as the cloud microphysics scheme; the Kain-Fritsch (new Eta) scheme was used as the cumulus parameterization scheme for the region. The $1.0 \times 1.0$ degree global tropospheric final (FNL) data provided by National Centers for Environmental Prediction (NCEP) were used to generate the initial field and lateral boundary conditions.

Hydrological Modeling. For a large area like Jinshajiang River Basin, its lack of historical data makes the distributed hydrological model inappropriate considering its computational efficiency and accuracy. Nevertheless, the lumped hydrological model is also not a suitable choice as it cannot distinguish the difference between sub-basins on their properties of runoff generation and flow routing. Therefore, we took a balanced approach that divided the Jinshajiang River Basin into several sub-basins and developed hydrological model respectively. The Xinanjiang model, which has proven to be very effective in humid areas, was adopted in this study for runoff generation[8]. The Muskingum channel routing method was adopted for flow routing to obtain hydrographs at specific locations of a basin.

\section{Application}

We examine the suitable parameterization and nesting mode of the WRF model over the Jinshajiang River Basin firstly, and then establish the hydrological model after calibration and validation, and analyze the coupled hydro-meteorological system in the end.

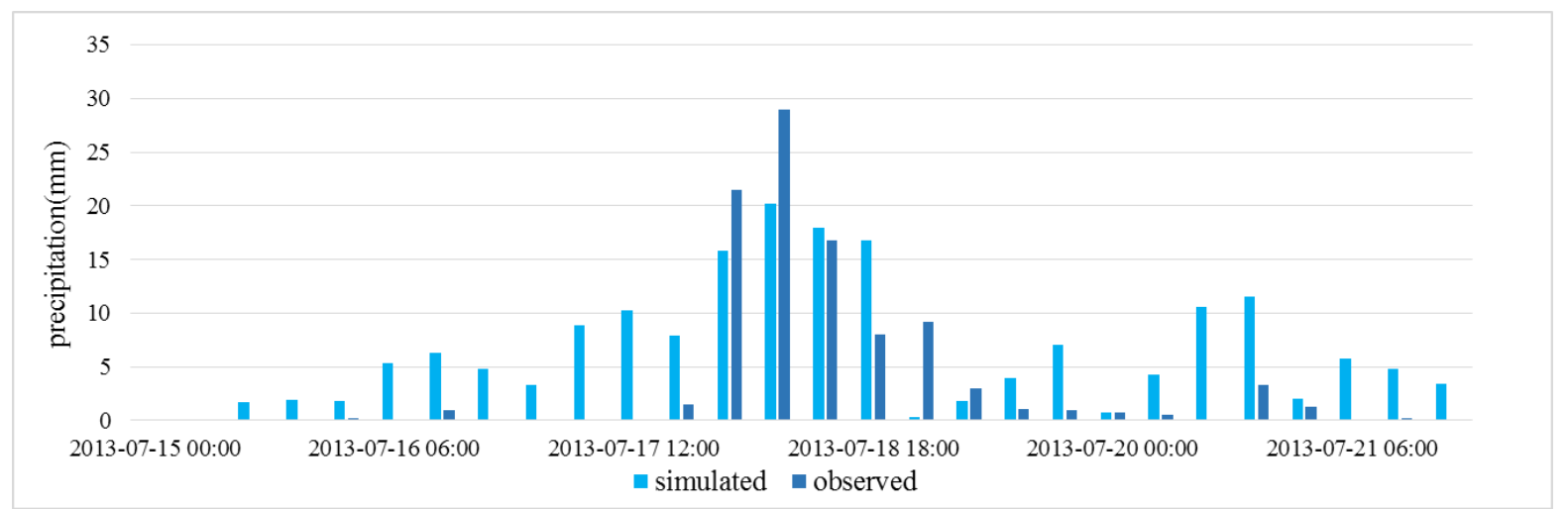

Fig. 2 The simulated precipitation of Xiluodu section by the WRF model

As can be seen from the Fig. 2, the simulated precipitation of every 6 hours in Xiluodu section by the WRF model does describe the precipitation process as it actually occurred, although the forecast value is bigger than the observed value sometimes. For the extreme precipitation event between July 17, 2013 and July 19, 2013, the simulated precipitation achieves its max value and this will be of great significance when it is used as the input of the hydrological model. 


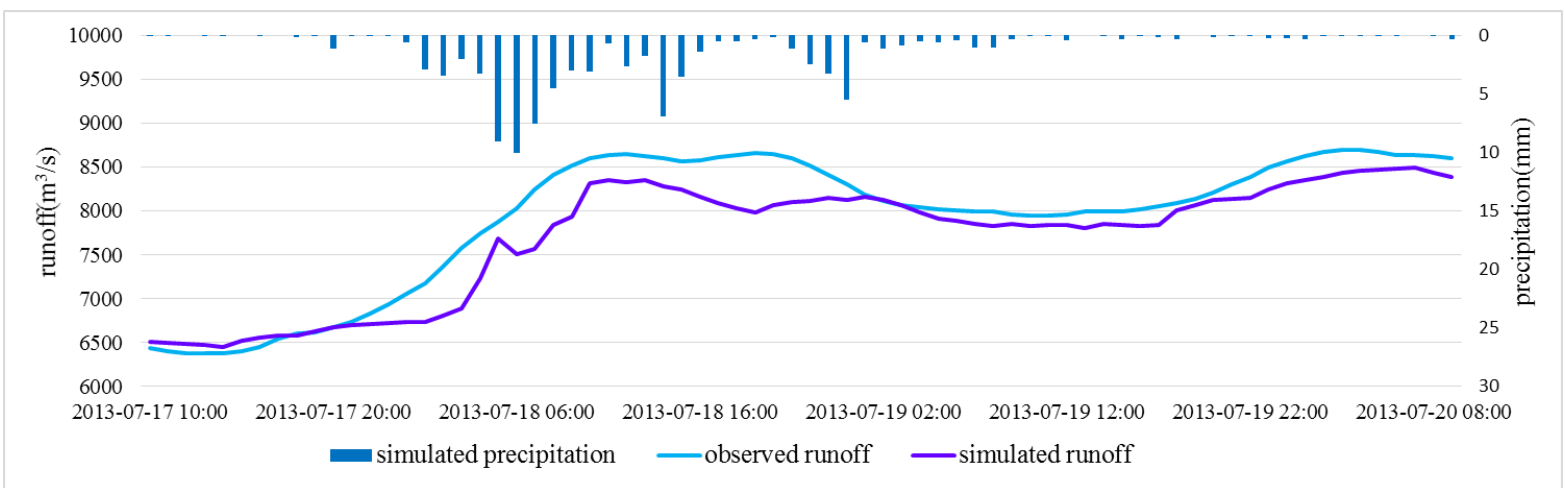

Fig. 3 The simulated runoff of Xiluodu section by the hydro-meteorological model

Fig. 3 shows the simulated runoff of Xiluodu section by the established one-way coupled hydro-meteorological model. The hydrograph indicates that the runoff forecast has the ability to reflect reality as it can figure out the flood peak correctly in some degree. In general, the precipitation and runoff forecast by the hydro-meteorological model can be used as a guidance in the practical production of hydropower system.

\section{Conclusions}

In this study, the Jinshajiang River Basin, which is the largest hydropower base in China, was selected as the study area. The WRF model, known as a next-generation mesoscale numerical weather prediction model, was firstly investigated to find a suitable mesoscale weather model for precipitation simulation in the Jinshajiang River Basin. The results show that the WRF model could be used for precipitation simulation and prediction in the Jinshajiang River Basin. On the basis of the WRF model for precipitation in the Jinshajiang River Basin, a one-way coupled hydro-meteorological model was built and also achieved a good performance, which allows it to be used in hydrological forecasting operations to provide a forecast flood with high precision.

\section{Acknowledgements}

This work is supported by the State Key Program of National Natural Science Foundations of China (No. 51239004 and 91547208), the National Natural Science Foundation of China (No. 51579107, 51509095 and 51309105)

\section{References}

[1] Cao J, Hu J, Tao Y. Journal of Geophysical Research: Atmospheres (2012),117

[2] Zhang Q, Xu C, Zhang Z, et al. J HYDROL (2008),353:215-227

[3] Su B, Xiao B, Zhu D, Jiang T. Hydrological Sciences Journal (2005),50

[4] Hong S, Dudhia J, Chen S. MON WEATHER REV (2004),132:103-120

[5] Srivastava PK, Islam T, Gupta M, Petropoulos G, Dai Q. WATER RESOUR MANAG (2015),29:2267-2284

[6] Wu W, Xu S, Lu H, et al. J ASIAN EARTH SCI (2011),40:611-621

[7] Jankov I, Gallus Jr WA, Segal M, Shaw B, Koch SE. WEATHER FORECAST (2005),20:1048-1060

[8] Ren-Jun Z. J HYDROL (1992), 135:371-381 